\title{
Acute Megakaryoblastic Leukemia with NUP98-KDM5A
}

National Cancer Institute

\section{Source}

National Cancer Institute. Acute Megakaryoblastic Leukemia with NUP98-KDM5A. NCI

Thesaurus. Code C132111.

A non-Down syndrome acute megakaryoblastic leukemia that occurs in childhood. It is associated with $\mathrm{t}(11 ; 12)(\mathrm{p} 15 ; \mathrm{p} 13)$ which results in the presence of NUP98-KDM5A

chimeric oncogene. 